\title{
HINWEISE UND VERZEICHNISSE
}

\section{Abgekürzt zitierte Literatur (Bibliografie)}

Die Abkürzungen und Siglen folgen den Richtlinien des Österreichischen Archäologischen Instituts $<\mathrm{http} / /$ www.oeai.at/publik/autoren.html>.

Alzinger 1962

Alzinger 1970

Alzinger 1972-1975

Alzinger 1974

Atlante I

Atlante II
W. Alzinger, Die Stadt des siebenten Weltwunders. Die Wiederentdeckung von Ephesos (Wien 1962).

RE Suppl. XII (1970) 1588-1704 s. v. Ephesos: B. Archäologischer Teil (W. Alzinger).

W. Alzinger, Das Regierungsviertel, ÖJh 50, 1972-1975, Beibl. 229-300.

W. Alzinger, Augusteische Architektur in Ephesos, SoSchrÖAI 16 (Wien 1974).

Enciclopedia dell'Arte Antica. Classica e Orientale. Atlante delle Forme Ceramiche I. Ceramica fine Romana nel Bacino Mediterraneo (Roma 1981).

Enciclopedia dell'Arte Antica. Classica e Orientale. Atlante delle Forme Ceramiche II. Ceramica fine Romana nel Bacino Mediterraneo (Roma 1985).

Aurenhammer (in Druckvorbereitung) M. Aurenhammer (Hrsg.), Die Skulpturen von Ephesos. Die Porträts, FiE 10, 3 (in Druckvorbereitung)

Aurenhammer - Sokolicek (in Druck) M. Aurenhammer - A. Sokolicek, The Remains of the Centuries. Sculptures and StatueBases in Late Antique Ephesus. The Evidence of the Upper Agora, in: Ch. Ratté - O. Dally (Hrsg.), Archaeology and the Cities of Asia Minor in Late Antiquity. An International Symposium at the University of Michigan, 8.-10. Jänner 2008, JRA Suppl. (in Druck).

Aydin 2007 B. Aydın, The Hellenistic Pottery and Small Finds of Kordon Tumulus at Kordon Köyü/Salihli (Manisa) 2001, ÖJh 76, 2007, 7-63.

Bammer 1976/1977

Bammer 2008

A. Bammer, Zu ÖJh 50, 1972-1975 Beibl. 242 ff. 249 ff., ÖJh 51, 1976/1977, $57-58$.

A. Bammer, Zur Dekonstruktion römischer Architektur. Studien zur Architektur im Nordbereich der sog. Oberen Agora von Ephesos, Anatolia Antiqua 16, 2008, 165-180.

Bammer - Fleischer - Knibbe 1974 A. Bammer - R. Fleischer - D. Knibbe, Führer durch das Archäologische Museum in Selçuk-Ephesos (Wien 1974).

Bauer 1996

F. A. Bauer, Stadt, Platz und Denkmal in der Spätantike. Untersuchungen zur Ausstattung des öffentlichen Raums in den spätantiken Städten Rom, Konstantinopel und Ephesos (Mainz 1996).

Berns - Hesberg - Vandeput - Waelkens 2002 Ch. Berns - H. v. Hesberg - L. Vandeput - M. Waelkens (Hrsg.), Patris und Imperium. Kulturelle und politische Identität in den Städten der römischen Provinzen Kleinasiens in der frühen Kaiserzeit. Kolloquium Köln, November 1998, BABesch Suppl. 8 (Leiden 2002).

Bier (in Druck)

Brands - Severin 2003

L. Bier, The Bouleuterion at Ephesus, FiE 9, 5 (in Druck).

G. Brands - H.-G. Severin (Hrsg.), Die spätantike Stadt und ihre Christianisierung. Symposium vom 14. bis 16. Februar 2000 in Halle/Saale (Wiesbaden 2003).

Brandt - Gassner - Ladstätter 2005 B. Brandt - V. Gassner - S. Ladstätter (Hrsg.), Synergia. Festschrift Friedrich Krinzinger I (Wien 2005).

Consp.

E. Ettlinger - B. Hedinger - B. Hoffmann - Ph. M. Kenrick - G. Pucci - K. Roth-Rubi G. Schneider - S. von Schnurbein - C. M. Wells - S. Zabehlicky-Scheffenegger, Conspectus formarum terrae sigillatae italico modo confectae, Materialien zur römisch-germanischen Keramik 10 (Bonn 1990).

Cook 1959/1960

Eichler 1961

J. M. Cook, Greek Archaeology in Western Asia Minor, ARepLond 1959/60, 27-57.

F. Eichler, Die österreichischen Ausgrabungen in Ephesos im Jahre 1960, AnzWien 98, 1961, 65-75.

Eichler 1962

F. Eichler, Die österreichischen Ausgrabungen in Ephesos im Jahre 1961, AnzWien 99, 1962, 37-54.

Eichler 1963

Eichler 1964
F. Eichler, Die österreichischen Ausgrabungen in Ephesos im Jahre 1962, AnzWien 100, 1963, 45-60.

F. Eichler, Die österreichischen Ausgrabungen in Ephesos im Jahre 1963, AnzWien 101, 1964, 39-44. 
Eichler 1965

Engelmann 1985

Fenn 2008

Fittschen 1973

Fleischer 1973

Fleischer 1978

Fleischer 1983

Fleischer 1999

Fleischer 2002

Fleischer 2008

Foss 1979

Frazer 1885

Friesinger - Krinzinger 1999

Gassner 1997

Graf 1999

Grierson

Groh 2006

Hagemann 1880

Halfmann 2001

Hansen - Fischer-Hansen 1994

Hayes 1972

Herda 2005

Hoepfner - Lehmann 2006

Hoepfner - Schwandner 1994

Holland 1944

Humann 1904

İnan - Rosenbaum 1966

Iro - Schwaiger - Waldner 2009

Jobst 1977

Karwiese 1995

Karwiese 1999
F. Eichler, Die österreichischen Ausgrabungen in Ephesos im Jahre 1964, AnzWien 102, 1965, 93-110.

H. Engelmann, Die Bauinschriften des Prytaneions von Ephesos, in: Lebendige Altertumswissenschaft. Festschrift Herrmann Vetters (Wien 1985) 155-157.

N. Fenn, Thin-Walled Pottery from an Early Imperial Complex at Priene/Ionia, ReiCretActa 40, 2008, 249-254.

K. Fittschen, Rez. zu: »J. İnan - E. Rosenbaum, Roman and Early Byzantine Portrait Sculpture in Asia Minor«, GGA 225, 1973, 46-67.

R. Fleischer, Artemis von Ephesos und verwandte Kultstatuen aus Anatolien und Syrien, EPRO 35 (Leiden 1973).

R. Fleischer, Artemis von Ephesos und verwandte Kultstatuen aus Anatolien und Syrien, in: S. Şahin - E. Schwertheim - J. Wagner (Hrsg.), Studien zur Religion und Kultur Kleinasiens. Festschrift Friedrich Karl Dörner, EPRO 66, 1 (Leiden 1978) 324-358.

R. Fleischer, Neues zu kleinasiatischen Kultstatuen, AA 1983, 81-93.

R. Fleischer, Neues zum Kultbild der Artemis von Ephesos, in: Friesinger - Krinzinger 1999, 605-609.

R. Fleischer, Die Amazonen und das Asyl des Artemisions von Ephesos, JdI 117, 2002, 185-216.

R. Fleischer, Die Kultstatue der Artemis von Ephesos und verwandte Götterbilder, in: Seipel 2008, 25-41.

C. Foss, Ephesus after Antiquity: A Late Antique, Byzantine and Turkish City (Cambridge 1979).

J. G. Frazer, The Prytaneum, the Temple of Vesta, the Vestals, Perpetual Fires, The Journal of Philology 14, 1885, 145-172.

H. Friesinger - F. Krinzinger (Hrsg.), 100 Jahre Österreichische Forschungen in Ephesos. Akten des Symposions Wien 1995, AForsch 1 = DenkschrWien 260 (Wien 1999).

V. Gassner, Das Südtor der Tetragonos-Agora. Keramik und Kleinfunde, FiE 13, 1, 1 (Wien 1997).

F. Graf, Ephesische und andere Kureten, in: Friesinger - Krinzinger 1999, 255-262.

$\mathrm{Ph}$. Grierson, Catalogue of the Byzantine Coins in the Dumbarton Oaks Collection and in the Whittemore Collection, III: Leo III. to Nicephorus III. 717-1081, Part 1: Leo III. to Michael III. (717-867) (Washington 1973).

St. Groh unter Mitarbeit von V. Lindinger - K. Löcker - W. Neubauer - S. S. Seren, Neue Forschungen zur Stadtplanung in Ephesos, ÖJh 75, 2006, 47-116.

G. Hagemann, De Prytaneo (Breslau 1880).

H. Halfmann, Städtebau und Bauherren im römischen Kleinasien. Ein Vergleich zwischen Pergamon und Ephesos, IstMitt Beih. 43 (Tübingen 2001).

M. H. Hansen - T. Fischer-Hansen, Monumental Political Architecture in Archaic and Classical Greek Poleis. Evidence and Historical Significance, in: D. Whitehead (Hrsg.), From Political Architecture to Stephanus Byzantius. Sources for the Ancient Greek Polis, Historia Einzelschriften 87 (Stuttgart 1994) 23-90.

J. W. Hayes, Late Roman Pottery (London 1972).

A. Herda, Apollon Delphinios, das Prytaneion und die Agora von Milet. Neue Forschungen, AA 2005, 243-294.

W. Hoepfner - L. Lehmann (Hrsg.), Die griechische Agora. Bericht über ein Kolloquium am 16. März 2003 in Berlin (Mainz 2006).

W. Hoepfner - E.-L. Schwandner, Haus und Stadt im klassischen Griechenland, Wohnen in der klassischen Polis I ${ }^{2}$ (München 1994).

L. B. Holland, Colophon, Hesperia 13, 1944, 91-171.

C. Humann, Magnesia am Maeander. Bericht über die Ergebnisse der Ausgrabungen der Jahre 1891-1893 (Berlin 1904).

J. İnan - E. Rosenbaum, Roman and Early Byzantine Portrait Sculpture in Asia Minor (London 1966).

D. Iro - H. Schwaiger - A. Waldner, Die Grabungen des Jahres 2005 in der Süd- und Nordhalle der Kuretenstraße, in: Ladstätter 2009, 53-87.

W. Jobst, Römische Mosaiken aus Ephesos I. Die Hanghäuser des Embolos, FiE 8, 2 (Wien 1977).

St. Karwiese, Groß ist die Artemis von Ephesos. Die Geschichte einer der großen Städte der Antike (Wien 1995).

St. Karwiese, Gedanken zur Entstehung des römischen Ephesos, in: Friesinger - Krinzinger 1999, 393-398. 
Keil 1939

\section{Kenzler 1999}

Kleiner 1968

Knell 1991

Knibbe 1981

Knibbe 1995

Knibbe 1998

Knibbe 1999

Knibbe - Langmann 1993

Knibbe - Thür 1995

Koester 1995

Krinzinger 2001

Krinzinger 2002

Ladstätter 2003

Ladstätter 2005

Ladstätter 2007

Ladstätter 2008

Ladstätter 2009

Ladstätter 2010

Ladstätter - Pülz 2007

Ladstätter - Sauer 2005

Ladstätter - Scheibelreiter 2010

Lätzer (in Druck)

Lang 1985

Lawall 2007

LiDonnici 1992

Liebeschuetz 2001

Martin 2003

Martin 2006
J. Keil, Kulte im Prytaneion von Ephesos, in: W. M. Calder - J. Keil (Hrsg.), Anatolian Studies Presented to William Hepburn Buckler (Manchester 1939) 119-128.

U. Kenzler, Studien zur Entwicklung und Struktur der griechischen Agora in archaischer und klassischer Zeit, Europäische Hochschulschriften 72 (Frankfurt 1999).

G. Kleiner, Die Ruinen von Milet (Berlin 1968).

H. Knell, Vitruvs Architekturtheorie. Versuch einer Interpretation ${ }^{2}$ (Darmstadt 1991).

D. Knibbe, Der Staatsmarkt. Die Inschriften des Prytaneions, FiE 9, 1, 1 (Wien 1981).

D. Knibbe, Via Sacra Ephesiaca: New Aspects of the Cult of Artemis Ephesia, in: Koester 1995, 141-155.

D. Knibbe, Ephesus. ЕФЕ $\Sigma \mathrm{O} \Sigma$. Geschichte einer bedeutenden antiken Stadt und Portrait einer modernen Großgrabung im 102. Jahr der Wiederkehr des Beginnes österreichischer Forschungen (1895-1997) (Frankfurt 1998).

D. Knibbe, Via Sacra Ephesiaca, in: Friesinger - Krinzinger 1999, 449-454.

D. Knibbe - G. Langmann, Via Sacra Ephesiaca I, BerMatÖAI 3 (Wien 1993).

D. Knibbe - H. Thür, Via Sacra Ephesiaca II. Grabungen und Forschungen 1992 und 1993 , BerMatÖAI 6 (Wien 1995).

H. Koester (Hrsg.), Ephesos. Metropolis of Asia. An Interdisciplinary Approach to its Archaeology, Religion, and Culture, Harvard Theological Studies 41 (Valley Forge 1995).

F. Krinzinger (Hrsg.), Studien zur hellenistischen Keramik in Ephesos, ÖJh Ergh. 2 (Wien 2001).

F. Krinzinger (Hrsg.), Das Hanghaus 2 von Ephesos. Studien zu Baugeschichte und Chronologie, AForsch 7 = DenkschrWien 302 (Wien 2002).

S. Ladstätter, Keramik, in: C. Lang-Auinger (Hrsg.), Hanghaus 1 in Ephesos. Funde und Ausstattung, FiE 8, 4 (Wien 2003) 22-85.

S. Ladstätter, Keramik, in: Thür 2005, 230-358.

S. Ladstätter, Mode oder politisches Manifest? Überlegungen zur Übernahme römischen Formenguts in der frühkaiserzeitlichen Keramik von Ephesos, in: Meyer 2007, 203-219.

S. Ladstätter, Römische, spätantike und byzantinische Keramik, in: Steskal - La Torre 2008, 97-189.

S. Ladstätter (Hrsg.), Neue Forschungen zur Kuretenstraße von Ephesos. Akten des Symposiums für Hilke Thür vom 13. Dezember 2006 an der Österreichischen Akademie der Wissenschaften in Wien, AForsch 15 = DenkschrWien 382 (Wien 2009).

S. Ladstätter, Wohneinheiten 1 und 2. Keramik, in: F. Krinzinger (Hrsg.), Hanghaus 2 in Ephesos. Die Wohneinheiten 1 und 2. Baubefund, Ausstattung, Funde, FiE 8, 8 (Wien 2010) 172-279. 530-587.

S. Ladstätter - A. Pülz, Ephesus in the Late Roman and Early Byzantine Period: Changes in its Urban Character from the Third to the Seventh Century AD, in: A. G. Poulter (Hrsg.), The Transition to Late Antiquity on the Danube and Beyond, Proceedings of the British Academy 141 (Oxford 2007) 391-433.

S. Ladstätter - R. Sauer, Late Roman C Ware und lokale spätantike Feinware aus Ephesos, in: F. Krinzinger (Hrsg.), Spätantike und mittelalterliche Keramik aus Ephesos, AForsch $13=$ DenkschrWien 332 (Wien 2005) 143-210.

S. Ladstätter - V. Scheibelreiter (Hrsg.), Städtisches Wohnen im östlichen Mittelmeerraum. 4. Jh. v. Chr. - 1. Jh. n. Chr. Akten des internationalen Kolloquiums vom 24.-27. Oktober 2007 an der Österreichischen Akademie der Wissenschaften, AForsch $18=$ DenkschrWien 397 (Wien 2010).

A. Lätzer, Studien zu einem späthellenistisch-frührömischen Fundkomplex aus dem Hanghaus 2, ÖJh 78, 2009 (in Druck).

G. J. Lang, Zur oberen Osthalle der Agora, der »Neronischen Halle« in Ephesos, in: Lebendige Altertumswissenschaft. Festschrift Hermann Vetters (Wien 1985) 176-180.

M. Lawall, Hellenistic Stamped Amphora Handles, in: Mitsopoulos-Leon - Lang-Auinger 2007, 28-60.

L. R. LiDonnici, The Images of Artemis Ephesia and Greco-Roman Worship. A Reconsideration, HarvTheoIR 85, 1992, 389-415.

J. H. W. G. Liebeschuetz, The Decline and Fall of the Roman City (Oxford 2001).

A. Martin, Western Sigillata in the Hanghäuser at Ephesos, ReiCretActa 38, 2003, 247-249.

A. Martin, Italian Sigillata in the East: Two Different Models of Supply (Ephesos and Ostia), in: D. Malfitana - J. Poblome - J. Lund (Hrsg.), Old Pottery in a New Century. Innovating Perspectives on Roman Pottery Studies. Atti del Convegno Internazionale di Studi Catania, 22-24 Aprile 2004 (Catania 2006) 175-188. 
McDonald 1943

McDonald 1948

Meriç 2002

Merkelbach 1980

Meyer 2007

Meyer-Schlichtmann 1988

Miller 1978

Miltner 1956-1958

Miltner 1957

Miltner 1958a

Miltner 1958b

Miltner 1959

Mitsopoulos-Leon 1972-1975

Mitsopoulos-Leon 1991

Mitsopoulos-Leon 2007

Mitsopoulos-Leon - Lang-Auinger 200

Morris 2001

Murray 1990

OCK

Peacock - Williams

Plattner 2003

Plattner 2008

Plattner 2010

Portefaix 1999

Preuner 1864

Quatember - Waldner - Pfisterer - Aurenhammer 2008 U. Quatember - A. Waldner - M. Pfisterer - M. Aurenhammer, Die

Rogers 1991

Rogers 1999a

Rogers 1999b

Rogers 2007

Rotroff 1997

Rotroff 2006

W. A. McDonald, The Political Meeting Places of the Greeks (Baltimore 1943).

W. A. McDonald, Types of Greek Civic Architecture - The Prytaneion, AJA 52, 1948, $374 \mathrm{f}$.

R. Meriç, Späthellenistisch-römische Keramik und Kleinfunde aus einem Schachtbrunnen am Staatsmarkt in Ephesos, FiE 9, 3 (Wien 2002).

R. Merkelbach, Der Kult der Hestia im Prytaneion der griechischen Städte, ZPE 37, 1980, 77-92.

M. Meyer (Hrsg.), Neue Zeiten - Neue Sitten. Zu Rezeption und Integration römischen und italischen Kulturguts in Kleinasien. Akten des Internationalen Kolloquiums in Wien 2005, WForsch 12 (Wien 2007).

C. Meyer-Schlichtmann, Die pergamenische Sigillata aus der Stadtgrabung von Pergamon. Mitte 2. Jh. v. Chr. - Mitte 2. Jh. n. Chr., PF 6 (Berlin 1988).

St. G. Miller, The Prytaneion. Its Function and Architectural Form (Berkeley 1978).

F. Miltner, XXI. Vorläufiger Bericht über die Ausgrabungen in Ephesos, ÖJh 43, 1956-1958, Beibl. 27-36.

F. Miltner, Ergebnisse der österreichischen Ausgrabungen in Ephesos im Jahre 1956, AnzWien 94, 1957, 13-25.

F. Miltner, Ephesos. Stadt der Artemis und des Johannes (Wien 1958).

F. Miltner, Die neuen Artemisstatuen aus Ephesos, Anatolia 3, 1958, 21-34.

F. Miltner, XXII. Vorläufiger Bericht über die Ausgrabungen in Ephesos, ÖJh 44, 1959, Beibl. 289-312.

V. Mitsopoulos-Leon, Keramik aus Basilika und Prytaneion - Ein Überblick, ÖJh 50, 1972-1975, Beibl. 495-524.

V. Mitsopoulos-Leon, Die Basilika am Staatsmarkt in Ephesos. Kleinfunde. 1. Teil: Keramik hellenistischer und römischer Zeit, FiE 9, 2, 2 (Wien 1991).

V. Mitsopoulos, Die Lampen, in: Mitsopoulos-Leon - Lang-Auinger 2007, 64-113.

V. Mitsopoulos-Leon - C. Lang-Auinger (Hrsg.), Die Basilika am Staatsmarkt in Ephesos. 2. Teil: Funde klassischer bis römischer Zeit, FiE 9, 2, 3 (Wien 2007).

S. P. Morris, The Prehistoric Background of Artemis Ephesia: A Solution to the Enigma of her >Breasts`?, in: U. Muss (Hrsg.), Der Kosmos der Artemis von Ephesos, SoSchrÖAI 37 (Wien 2001) 135-151.

O. Murray (Hrsg.), Sympotica. A Symposium on the Symposion (Oxford 1990).

A. Oxé - H. Comfort - Ph. Kenrick, Corpus Vasorum Arretinorum: A Catalogue of the Signatures, Shapes and Chronology of Italian Sigillata, Antiquitas 41 (Bonn 2000).

D. Peacock - D. Williams, Amphorae and the Roman Economy: An Introductory Guide (London 1986).

G. A. Plattner, Ephesische Kapitelle des 1. und 2. Jhs. n. Chr. (ungedr. Diss. Universität Wien 2003).

G. A. Plattner, Bauornamentik, in: Steskal - La Torre 2008, 275-282.

G. A. Plattner, Architekturdekoration, in: F. Krinzinger (Hrsg.), Hanghaus 2 in Ephesos. Die Wohneinheiten 1 und 2. Baubefund, Ausstattung, Funde, FiE 8, 8 (Wien 2010) 149-155. 510-524.

L. Portefaix, The Image of Artemis Ephesia - A Symbolic Configuration Related to Her Mysteries?, in: Friesinger - Krinzinger 1999, 611-617.

A. Preuner, Hestia-Vesta. Ein Cyclus religionsgeschichtlicher Forschungen (Tübingen 1864).

Grabung des Jahres 2005 beim Nymphaeum Traiani in Ephesos, ÖJh 77, 2008, 265-334.

G. M. Rogers, The Sacred Identity of Ephesos. Foundation Myths of a Roman City (London 1991).

G. M. Rogers, The Mysteries of Artemis at Ephesos, in: Friesinger - Krinzinger 1999, 241-250

G. M. Rogers, The Philosebastoi Kuretes of Ephesos, in: Scherrer - Taeuber - Thür 1999, $125-130$

G. M. Rogers, From the Greek Polis to the Graeco-Roman Polis. Augustus and the Artemision of Ephesos, in: H. Elton - G. Reger (Hrsg.), Regionalism in Hellenistic and Roman Asia Minor. Acts of the Conference Hartford, Connecticut (USA), August 22-24 1997 (Bordeaux 2007) 137-145.

S. Rotroff, Hellenistic Pottery. Athenian and Imported Wheelmade Table Ware and Related Material, Agora 29 (Athen 1997).

S. Rotroff, Hellenistic Pottery: The Plain Wares, Agora 33 (Athen 2006). 
Rumscheid 1998

Scherrer 1990

Scherrer 1995a

Scherrer 1995b

Scherrer 1997

Scherrer 2007

Scherrer - Taeuber - Thür 1999

Schmitt Pantel 1992

Schwarzer 2004

Schwarzer 2008

Seipel 2008

Seiterle 1979

SNG Tüb.

Soldan 1999

Steskal 2007

Steskal 2008

Steskal - La Torre 2008

Thür 1995

Thür 1996

Thür 1999

Thür 2003

Thür 2005

Thür 2007

Tosi 1966

Turnovsky 2005a

Turnovsky 2005b

Vaag - Nørskov - Lund 2002
F. Rumscheid, Priene. Führer durch das »Pompeji Kleinasiens« (Istanbul 1998).

P. Scherrer, Augustus, die Mission des Vedius Pollio und die Artemis Ephesia, ÖJh 60, 1990, 87-101.

P. Scherrer (Hrsg.), Ephesos. Der neue Führer. 100 Jahre österreichische Ausgrabungen 1895-1995 (Wien 1995).

P. Scherrer, The City of Ephesos from the Roman Period to Late Antiquity, in: Koester 1995, 1-25.

P. Scherrer, Anmerkungen zum städtischen und provinzialen Kaiserkult: Paradigma Ephesos - Entwicklungslinien von Augustus bis Hadrian, in: H. Thür (Hrsg.), »... und verschönerte die Stadt ...«. ... KAI KO $\Sigma$ MH $\Sigma$ ANTA THN ПO $\Lambda$ IN ... Ein ephesischer Priester des Kaiserkultes in seinem Umfeld, SoSchrÖAI 27 (Wien 1997) 93-112.

P. Scherrer, Der conventus civium Romanorum und kaiserliche Freigelassene als Bauherren in Ephesos in augusteischer Zeit, in: Meyer 2007, 63-75.

P. Scherrer - H. Taeuber - H. Thür (Hrsg.), Steine und Wege. Festschrift Dieter Knibbe, SoSchrÖAI 32 (Wien 1999).

P. Schmitt Pantel, La cité au banquet. Histoire des repas publics dans les cités grecques, CEFR 157 (Rom 1992).

H. Schwarzer, Der sogenannte Bau H. Zum mutmaßlichen Prytaneion von Pergamon, IstMitt 54, 2004, 173-182.

H. Schwarzer, Die Stadtgrabung. Das Gebäude mit dem Podiensaal in der Stadtgrabung von Pergamon, AvP 15, 4 (Berlin 2008).

W. Seipel (Hrsg.), Das Artemision von Ephesos. Heiliger Platz einer Göttin. Eine Ausstellung des Kunsthistorischen Museums Wien in Zusammenarbeit mit dem Archäologischen Museum Istanbul und dem Ephesos-Museum, Selçuk. Archäologisches Museum Istanbul, 22. Mai bis 22. September 2008 (Wien 2008).

G. Seiterle, Artemis, die große Göttin von Ephesos. Eine neue Deutung der Vielbrüstigkeit eröffnet einen Zugang zum bisher unbekannten Kult der Göttin, AW 10, 3, 1979, 3-16.

Sylloge Nummorum Graecorum. Deutschland. Münzsammlung der Universität Tübingen, 4. Heft: Mysien - Ionien (München 1989).

U. Soldan, Frauen als Funktionsträgerinnen im kaiserzeitlichen Ephesos: Die weiblichen Prytaneis, in: Friesinger - Krinzinger 1999, 115-119.

M. Steskal, Konstruktionszeichnungen zweier Voluten aus dem Prytaneion in Ephesos, ÖJh 76, 2007, 371-392.

M. Steskal, Rituelle Bestattungen im Prytaneion von Ephesos? Zu den Fundumständen der Artemis Ephesia-Statuen, ÖJh 77, 2008, 363-373.

M. Steskal - M. La Torre, Das Vediusgymnasium in Ephesos. Achäologie und Baubefund, FiE 14, 1 (Wien 2008).

H. Thür, Der ephesische Ktistes Androklos und (s)ein Heroon am Embolos, ÖJh 64, 1995, 63-103.

H. Thür, Ein dorischer Torbau auf dem Staatsmarkt in Ephesos, in: F. Blakolmer K. R. Krierer - F. Krinzinger - A. Landskron-Dinstl - H. D. Szemethy - K. Zhuber-Okrog (Hrsg.), Fremde Zeiten. Festschrift Jürgen Borchhardt I (Wien 1996) 345-361.

H. Thür, Die spätantike Bauphase der Kuretenstraße, in: R. Pillinger - O. Kresten - F. Krinzinger - E. Russo (Hrsg.), Efeso paleocristiana e bizantina - Frühchristliches und byzantinisches Ephesos, AForsch 3 = DenkschrWien 282 (Wien 1999) 104-120.

H. Thür, Das spätantike Ephesos. Aspekte zur Frage der Christianisierung des Stadtbildes, in: Brands - Severin 2003, 259-273.

H. Thür (Hrsg.), Hanghaus 2 in Ephesos. Die Wohneinheit 4. Baubefund, Ausstattung, Funde, FiE 8, 6 (Wien 2005).

H. Thür, Wie römisch ist der sog. Staatsmarkt in Ephesos?, in: Meyer 2007, 77-90.

G. Tosi, Contributo allo studio dei pritanei, ArtAntMod 33, 1966, 10-21. 151-172.

P. Turnovsky, Late Antique and Byzantine Pottery of the Church of St. Mary in Ephesos. An Introduction, ReiCretActa 39, 2005, 217-224.

P. Turnovsky, The Morphological Repertory of Late Roman / Early Byzyantine Coarse Wares in Ephesos, in: J. M. Gurt I Esparraguera - J. Buxeda I Garrigós - M. A. Cau Ontiveros, LRCW I. Late Roman Coarse Wares, Cooking Wares and Amphorae in the Mediterranean, BARIntSer 1340 (Oxford 2005) 635-645.

L. E. Vaag - V. Nørskov - J. Lund, The Pottery. Ceramic Material and Other Finds from Selected Contexts, The Maussolleion at Halikarnassos, Reports of the Danish Archaeological Expedition to Bodrum 7 (Aarhus 2002). 
Waldner 2009a

Waldner 2009b

Warner Slane 1997

Wilberg 1923

Witetschek 2008
A. Waldner, Keramische Evidenzen zur Baugeschichte des unteren Embolos von Ephesos (ungedr. Diss. Universität Wien 2009).

A. Waldner, Heroon und Oktogon. Zur Datierung zweier Ehrenbauten am unteren Embolos von Ephesos anhand des keramischen Fundmaterials aus den Grabungen von 1989 und 1999, in: Ladstätter 2009, 283-315.

K. Warner Slane, The Fine Wares, in: S. Herbert (Hrsg.), Tel Anafa II, I, JRA Suppl. 10 (Ann Arbor 1997) 249-406.

W. Wilberg, Die Agora, in: FiE 3 (Wien 1923) 1-90.

St. Witetschek, Ephesische Enthüllungen 1. Frühe Christen in einer antiken Großstadt zugleich ein Beitrag zur Frage nach den Kontexten der Johannesapokalypse, Biblical Tools and Studies 6 (Leiden 2008).

Zabehlicky-Scheffenegger - Schneider 2005 S. Zabehlicky-Scheffenegger - G. Schneider, Ephesian Cooking Vessels of the Augustan Period, in: M. Berg Briese - L. E. Vaag (Hrsg.), Trade Relations in the Eastern Mediterranean from the Late Hellenistic Period to Late Antiquity: The Ceramic Evidence, Halicarnassian Studies 3 (Odense 2005) 63-67.

\section{Antike Autoren}

Die Abkürzungen der antiken Autoren und Werktitel folgen H. Cancik - H. Schneider (Hrsg.), Der Neue Pauly. Enzyklopädie der Antike (1996) I, A-Ari S. XXXIX-XLVII.

\section{Verzeichnis der im Text und Katalog verwendeten Abkürzungen}

Neben den vom Deutschen Archäologischen Institut vorgeschlagenen Abkürzungen und Kurzformen $<$ http:// www.dainst.org> werden folgende verwendet:

$\begin{array}{llll}\text { A. } & \text { Anfang } & \text { H. } & \text { Hälfte } \\ \text { AO } & \text { Aufbewahrungsort } & \text { hell. } & \text { Hellenistisch } \\ \text { ARS } & \text { African Red Slip Ware } & \text { inkl. } & \text { inklusive } \\ \text { B } & \text { Breite } & \text { InvNr. } & \text { Inventar-Nummer } \\ \text { Bdfrgt. } & \text { Bodenfragment(e) } & \text { ITS } & \text { Italische Sigillata } \\ \text { Bearb. } & \text { Bearbeitung } & \text { KatNr. } & \text { Katalog-Nummer } \\ \text { cf. } & \text { confer } & \text { L } & \text { Länge } \\ \text { D. } & \text { Drittel } & \text { Lit. } & \text { Literatur } \\ \text { Dm } & \text { Durchmesser } & \text { LRC } & \text { Late Roman C-Ware } \\ \text { DmBd } & \text { Durchmesser Boden } & \text { M. } & \text { Mitte } \\ \text { D } & \text { Dicke } & \text { Mat. } & \text { Material } \\ \text { DmRd } & \text { Durchmesser Rand } & \text { o. } & \text { oben } \\ \text { DmWd } & \text { Durchmesser Wand } & \text { o. Nr. } & \text { ohne Nummer } \\ \text { E. } & \text { Ende } & \text { Rd } & \text { Rand } \\ \text { EM } & \text { Efes Müzesi Selçuk } & \text { Rdfrgt. } & \text { Randfragment(e) } \\ \text { erh. } & \text { erhalten } & \text { rek. } & \text { rekonstruierte(r) } \\ \text { Erh. } & \text { Erhaltung } & \text { RL } & \text { Rüstloch } \\ \text { ERSW } & \text { Ephesian Red Slip Ware } & \text { ROW-Lampe } & \text { Red-on-White-Lampe } \\ \text { ESA } & \text { Eastern Sigillata A } & \text { s. } & \text { siehe } \\ \text { ESB } & \text { Eastern Sigillata B } & \text { SE } & \text { Stratigrafische Einheit } \\ \text { ESC } & \text { Eastern Sigillata C } & \text { T } & \text { Tiefe } \\ \text { FJ } & \text { Fundjahr } & \text { TR } & \text { Tonrohr } \\ \text { FO } & \text { Fundort } & \text { u. } & \text { unten } \\ \text { Frgt. } & \text { Fragment(e) } & \text { ungedr. } & \text { ungedruckte } \\ \text { Gew. } & \text { Gewicht } & \text { V. } & \text { Viertel } \\ \text { GHD } & \text { Grabungshaus-Depot Selçuk } & \text { Verf. } & \text { Verfasser } \\ \text { H } & \text { Höhe } & \text { Wdfrgt. } & \text { Wandfragment(e) }\end{array}$

Spezifische numismatische Abkürzungen sind am Beginn des relevanten Kapitels angeführt. 


\section{Abkürzungen der Katalogobjekte}

$\begin{array}{ll}\text { A } & \text { Architekturteile } \\ \text { M } & \text { Münzen } \\ \text { K } & \text { Hellenistische, römische und spätantike Keramik } \\ \text { SK } & \text { Skulptur }\end{array}$

\section{Alphabetisches Autorenverzeichnis}

Nicole M. High

Kelsey Museum of Archaeology, University of Michigan, 434 South State Street, Ann Arbor, MI 481091390, USA

Sabine Ladstätter

Österreichisches Archäologisches Institut

A-1190 Wien, Franz Klein-Gasse 1

Matthias Pfisterer

Kunsthistorisches Museum Wien, Münzkabinett

A-1010 Wien, Burgring 5

Georg A. Plattner

Kunsthistorisches Museum Wien, Antikensammlung

A-1010 Wien, Burgring 5

Martin Steskal

Österreichisches Archäologisches Institut

A-1190 Wien, Franz Klein-Gasse 1

\section{Planzeichner und -umzeichner, Fotografen}

Alzinger, W.

Durgut, F. und O.

Eitler, J.

Fleischer, R.

Gail, N.

High, N. M.

Hochleitner, A.

John, W.

Jost, J.

Knibbe, D.

Kurtze, Ch

Ladstätter, S.

La Torre, M.

Math, N.

Mayrhofer, P.

Plattner, G. A.

Stark, B.

Steskal, M.

Zabrana, L.
W. A.

F. O. D.

J. E.

R. F.

N. G.

N. H.

A. $\mathrm{H}$.

W. J.

J. J.

D. K.

C. K.

S. L.

M. L.

N. M.

P. M.

G. P.

B. S.

M. S.

L. Z. 


\section{Abbildungen (mit inkludiertem Abbildungsnachweis)}

TAFELABBILDUNGEN

Tafelabb.

Kapitel I

Taf. 1, 1:

Taf. 2, 1:

Taf. 3, 1:

Taf. 4, 1:

Taf. 4, 2:

Taf. 5,1 :

Taf. 5, 2:

Taf. 5, 3:

Kapitel II

Taf. 6, 1:

Taf. 6, 2:

Taf. 6, 3:

Taf. 7, 1:

Taf. 7, 2:

Taf. 8, 1:

Taf. 8, 2:

Taf. 8, 3:

Taf. 9, 1:

Taf. 9, 2:

Taf. 9, 3:

Taf. 10, 1:

Taf. 10, 2:

Taf. 10, 3:

Taf. 11, 1:

Taf. 11, 2:

Taf. 12, 1:

Taf. 12, 2:

Taf. 13, 1:

Taf. 13, 2:

Taf. 14, 1:

Taf. 14, 2:

Taf. 15, 1:

Taf. 15, 2:

Taf. 16, 1:

Taf. 17, 1:

Taf. 17, 2:

Taf. 18, 1:

Prytaneion. Sondage II (1960). Südprofil

Taf. 18, 2:

Taf. 19, 1:

Taf. 19, 2:

Taf. 19, 3:

Taf. 20, 1:

Taf. 20, 2:

Inhalt

Ephesos. Stadtplan

Prytaneion. Aufnahme vom Bülbüldağ

Prytaneion von Süden

Prytaneion von Norden

Prytaneion von Osten Prytaneion 1955. Grundriss

Freilegung von Raum 6

>Schöne Artemis` in Fundlage (Raum 5)

$>$ Schöne Artemis` (EM InvNr. 718)

২Kleine Artemis` (EM InvNr. 717)

$>$ Große Artemis` in Fundlage (Vorhof)

$>$ Große Artemis` (EM InvNr. 712)

Prytaneion 1956. Grundriss

$>$ Kuretenhalle` am unteren Embolos

Oberer >Staatsmarkt< 1957 aus Sondage II

Schrankenplatte in Fundlage

Prytaneion. Sondage IV (1960)
Oberer \Staatsmarkt` von Ephesos. Übersichtsplan

Prytaneion. Generalisierter Grundriss mit Raumnummern (1:250) geschnittene Bauteile dunkel, aufsichtige Bauteile hell

Oberer >Staatsmarkt< von Ephesos. Luftbild

Prytaneion 1955. Freilegung des $>$ Hestiasaales $<$ entlang der Westwand Prytaneion 1955. Freilegung des >Hestiasaales`entlang der Westwand

Prytaneion 1955. Freilegung des Stereobats der Vorhalle

Anastylose des südöstlichen Säulenschaftes (A 16) des >Hestiasaales`

Freilegung des westlichen Stylobats des Vorhofes

Freilegung der Südostecke des Stylobats des Vorhofes

Kopie der >Kleinen Artemis` (EM 231/56)

Spätantik/byzantinisches Mauerwerk in Raum 5 vor Abtragung

Freilegung der $>$ Kuretenhalle am unteren Embolos

Scholastikiatherme. Eingemauerte Säule und Kapitell aus dem Prytaneion

Nordwand des >Hestiasaales` vor Entfernung des Statuentorsos

Nordwand des >Hestiasaales` nach Entfernung des Statuentorsos

Zeichnerische Aufnahme der Nordwand des >Hestiasaales` 1960

Zeichnerische Aufnahme der Nordwand der Vorhalle 1960

Prytaneion. Generalisierter Grundriss mit Sondagenplan 1960-1963 (1:250) Prytaneion. Sondage I (1960). Ost- und Südprofil

Prytaneion. Sondage I und II (1960). Planum

Prytaneion - Bereich $>$ Akademiegasse $\prec$. Schrankenplatte $(\mathrm{P} / 60 / 55)$

Prytaneion - Bereich >Akademiegasse «. Sondage II (1960)

Prytaneion - Bereich >Akademiegasse . Sondage II (1960).

Prytaneion. Sondage IV (1960). Planum, Nord- und Ostprofil

Prytaneion. Sondage IV und V (1960). Planum und Ostprofil
Nachweis

C. K., (C) ÖAI

C. K., (C) ÖAI

L. Z., P. M., M. S., C ÖAI

F. O. D., (C) ÖAI

N. G., (C) ÖAI

M. S., (C) ÖAI

M. S., (C) ÖAI

M. S., (C) ÖAI

Pl. 407, (C) ÖAI

Pl. 405, C ÖAI

Miltner 1956-1958, Abb. 18.

Pl. 406, C ÖAI

Pl. 656, C ÖAI

Pl. 634, (C) ÖAI

1956 9a, 4, (C) ÖAI

1956 9a, 7, (C) ÖAI

(C) ÖAI

N. G., (C) ÖAI

N. G., (C) ÖAI

(c) ÖAI

(C) ÖAI

N. G., (C) ÖAI

Miltner 1959, Abb. 139.

Pl. 606, (C) ÖAI

A-W-OAI-N III 0225, (C) ÖAI

Pl. 353, C ÖAI

1957 333, 12, (C) ÖAI

1957 5, 12, C ÖAI

19563,13 , (C) ÖAI

1961 224, 6, (C) ÖAI

Ephesos 1960, Prytaneion,

Blatt 1 (W. A., D. K.), (C) ÖAI

Ephesos 1960, Prytaneion,

Blatt 52 (W. J.), (C) ÖAI

L. Z., M. S., P. M., C ÖAI

Ephesos 1960, Prytaneion, Blatt 23 (W. A.), C ÖAI

Ephesos 1960, Prytaneion, Blatt 27 und 27A (W. A.), (C) ÖAI

Ephesos 1960, Prytaneion, Blatt 32 (W. A.), C ÖAI

Ephesos 1960, Prytaneion, Blatt 55 (W. J., W. A.), (C ÖAI Ephesos 1960, Prytaneion, Blatt 55 (W. J., W. A.), (C) ÖAI Ephesos 1960, Prytaneion, Blatt 55 (W. J., W. A.), (C) ÖAI Ephesos 1960, Prytaneion, Blatt 49 (W. A.), C ÖAI 1960 236, 10, C ÖAI Ephesos 1960, Prytaneion, Blatt 57 (W. A.), (C ÖAI 
Taf. 20, 3:

Taf. 21, 1:

Taf. 21, 2:

Taf. 21, 3:

Taf. 22, 1:

Taf. 22, 2:

Taf. 22, 3:

Taf. 23, 1:

Taf. 23, 2:

Taf. 23, 3:

Taf. 23, 4:

Taf. 24, 1:

Taf. 24, 2:

Taf. 24, 3:

Taf. 24, 4:

Taf. 25, 1:

Taf. 25, 2:

Taf. 25, 3:

Taf. 26, 1:

Taf. 26, 2:

Taf. 26, 3:

Taf. 26, 4:

Taf. 27, 1:

Taf. 28, 1:

Taf. 28, 2:

Taf. 29, 1:

Taf. 29, 2:

Taf. 29, 3:

Kapitel III Taf. 30, 1:

Taf. 31, 1:

Taf. 32, 1:

Taf. 32, 2:

Taf. 33, 1:

Taf. 34, 1:

Taf. 35,1 :

Taf. 35, 2:

Taf. 36, 1:

Taf. 37, 1:

Taf. 37, 2:

Taf. 37, 3:

Taf. 37, 4:
Prytaneion. Sondage VIII (1961). Planum

Prytaneion. Sondage VIII (1961). Südprofil

Prytaneion. Sondage VIII (1961). Nordwand der Vorhalle

Prytaneion. Sondage X (1961). Planum

Prytaneion. Sondage XI (1961). Planum

Zeichnerische Aufnahme der Südwand des `Hestiasaales` 1961 - Westteil

Zeichnerische Aufnahme der Südwand des `Hestiasaales` 1961 - Ostteil

Prytaneion. Sondage XVI (1961). Ostprofil

Prytaneion. Sondage XVI (1961). Osthälfte Raum 4

Prytaneion. Sondage XVIII (1961). Eingang in `Hestiasaak (Tür 3)

Prytaneion. Lesbisches Kymation aus Sondage XVIII (?)

Prytaneion. Sondage XIX (1961). Zentrales Fundament im >Hestiasaal Prytaneion. Sondagen XXI und XXVI (1961). Planum

Prytaneion. Sondage XXI (1961). Westprofil

Prytaneion. Sondage XXIII (1961). Planum

Prytaneion. Sondage XXII (1961). Planum

Prytaneion. Sondage XXII (1961). Ostprofile

Prytaneion. Sondage XXII (1961). Südhälfte Raum 6

Prytaneion. Sondage XXV (1961). Planum

Prytaneion von Norden (1961) mit Sondagen XVIII, XIX, XXV, XXVI und XXXI

Prytaneion. Sondage XXIX (1961). Planum und Südprofil

Prytaneion. Architekturprobe des dorischen Gebälks der Vorhalle (1961) Prytaneion 1961. Grundriss

Prytaneion. Sondage XXXI Nordhälfte (1962). Planum und Ostprofil

Prytaneion. Sondage XXXI Südhälfte (1962). Planum und Ostprofil

Prytaneion. Rekonstruktion der dorischen Ordnung der Vorhalle (1964) Prytaneion. Rekonstruierte dorische Ordnung der Vorhalle (1968)

Prytaneion. Rekonstruktion der Vorhalle (W. J.)

Prytaneion. Grundriss Bauaufnahme (1:150)

Prytaneion. Generalisierter Grundriss mit Hauptmaßen (1:250)

Vorhof von Süden

Vorhof. Südwand (1:100)

Prytaneion. Südfront $(1: 100)$

Vorhof. Ostwand (1:100)

Stylobatblöcke der Westseite

Stylobatblöcke der Südostecke

Stylobatblöcke der Westseite und der Südostecke (1:50)

Vorhof. Östliche Regenrinne von Norden

Vorhof. Südliche Regenrinne von Osten

Vorhof. Westliche Regenrinne von Süden

Nord-Süd verlaufender Kanal unter östlichem Umgang
Ephesos 1961, Prytaneion, Blatt 15 (W. A.), (C) ÖAI Ephesos 1961, Prytaneion, Blatt 10 (W. A.), (C) ÖAI 1961 246, 5, C ÖAI Ephesos 1961, Prytaneion, Blatt 19 (W. A.), (C) ÖAI Ephesos 1961, Prytaneion, Blatt 18 (W. A.), (C) ÖAI Ephesos 1961, Prytaneion, Blatt 28 (W. J.), C ÖAI Ephesos 1961, Prytaneion, Blatt 28A (W. A.), (C) ÖAI Ephesos 1961, Prytaneion, Blatt 56 (W. A.), C ÖAI 1961256,9 , (C) ÖAI 1961 256, 7, (C) ÖAI Ephesos 1961, Prytaneion, Blatt 62 (W. J.), (C) ÖAI 1961 256, 3, (C) ÖAI Ephesos 1961, Prytaneion, Blatt 50 (W. A.), (C) ÖAI Ephesos 1961, Prytaneion, Blatt 49 (W. A.), C ÖAI Ephesos 1961, Prytaneion, Blatt 54 (W. A.), (C ÖAI Ephesos 1961, Prytaneion, Blatt 36 (W. J.), (C) ÖAI Ephesos 1961, Prytaneion, Blatt 36A (W. J.), (C) ÖAI 1961 255, 3, C ÖAI

Ephesos 1961, Prytaneion, Blatt 48 (W. A.), (C) ÖAI 1961 252, 6, C ÖAI

Ephesos 1961, Prytaneion, Blatt 59 (W. A.), (C) ÖAI 1961 268b, 3, (C ÖAI Eichler 1962, Abb. 1. Ephesos 1962, Prytaneion, Blatt 37 (W. A.), (C) ÖAI Ephesos 1962, Prytaneion, Blatt 37A (W. A.), (C) ÖAI 1964 413, 1, (C) ÖAI 1968 627, 2, (C) ÖAI Alzinger 1974, Taf. 34, 65a.

P. M., M. S., J. E., L. Z., (C) ÖAI L. Z., P. M., M. S., (C) ÖAI M. S., (C) ÖAI P. M., L. Z., (C) ÖAI P. M., L. Z., (C) ÖAI P. M., L. Z., (C) ÖAI M. S., (C) ÖAI M. S., (C) ÖAI J. E., L. Z., (C) ÖAI M. S., (C) ÖAI M. S., (C) ÖAI M. S., (C) ÖAI M. S., (C) ÖAI 
Taf. 38, 1: Taf. 39, 1-3: Taf. 40, 1-3: Taf. 41, 1. 2: Taf. 42, 1:

Taf. 42, 2-4: Taf. 43,1 :

Taf. 43, 2:

Taf. 44, 1:

Taf. 44, 2:

Taf. 45,1 :

Taf. 45, 2:

Taf. 45, 3:

Taf. 46, 1:

Taf. 46, 2:

Taf. 46, 3:

Taf. 46, 4:

Taf. 47, 1:

Taf. 47, 2:

Taf. 47, 3 :

Taf. 48, 1:

Taf. 49, 1:

Taf. 50, 1:

Taf. 50, 2:

Taf. 51, 1:

Taf. 52, 1:

Taf. 53, 1:

Taf. 53, 2:

Taf. 53, 3:

Taf. 54, 1:

Taf. 54, 2:

Taf. 54, 3:

Taf. 55, 1:

Taf. 55, 2:

Taf. 56, 1:

Taf. 56, 2:

Taf. 57, 1:

Taf. 57, 2:

Taf. 57, 3:

Taf. 58, 1:

Taf. 58, 2:

Taf. 58, 3:

Taf. 58, 4:

Taf. 59, 1:

Taf. 59, 2:

Taf. 59, 3:

Taf. 60, 1:

Taf. 60, 2:

Taf. 60, 3:

Taf. 60, 4:

Taf. 61, 1:

Taf. 61, 2:

Taf. 61, 3:

Taf. 62, 1:

Taf. 62, 2:

Taf. 62, 3:

Taf. 62, 4:

Taf. 63, 1:

Taf. 63, 2:
Sockel und Aufsatz Ehrenbasis SK 12. 13 (1:25)

Sockel Ehrenbasis SK 12

Aufsatz Ehrenbasis SK 13

Aufsatz Ehrenbasis SK 13

Aufsatz Ehrenbasis SK 14 (1:25)

Aufsatz Ehrenbasis SK 14

Fundament und Bothros im Zentrum des Vorhofes (1:50)

Fundament und Bothros von Süden

Fundament und Bothros von Osten

Detail Bothros

Vorhalle von Westen

Vorhalle von Osten

Vorhalle von Süden

Vorhalle. Stereobat der dorischen Ordnung

Vorhalle. Euthynterieschicht von Westen

Stylobatblock vor der westlichen Antenmauer (1:50)

Stylobatblock vor der westlichen Antenmauer

Vorhalle. Westliche Antenmauer von Osten

Vorhalle. Westliche Antenmauer von Süden

Vorhalle. Westliche Antenmauer von Westen

Vorhalle und Raum 6. Westwände (1:100)

Prytaneion. Westfront (1:125)

Vorhalle. Östliche Antenmauer von Westen

Vorhalle. Östliche Antenmauer von Süden

Vorhalle und >Hestiasaak . Ostwände (1:100)

Vorhalle. Nordwand (1:100)

Vorhalle. Nordwand im Bereich von Tür 1

Vorhalle. Nordwand im Bereich von Tür 2

Vorhalle. Nordwand im Bereich von Tür 3

Vorhalle. Nordwand im Bereich von Tür 4

Vorhalle. Abgemauerte Türen 1 und 2

Vorhalle. Sekundäre Türschwelle in Tür 4

Vorhalle. Kureteninschrift auf Nordwand (IvE 1023)

Vorhalle. Kureteninschrift auf Nordwand (IvE 1024)

Vorhalle. Dorische Ordnung von Süden (1:100)

Vorhalle. Dorische Ordnung von Norden (1:100)

Vorhalle. Dorische Ordnung. Draufsicht (1:50)

Vorhalle. Dorische Ordnung. Schnitt durch Gebälk nach Westen (1:50)

Vorhalle. Dorische Ordnung. Säulen A 3. A 4 (1:100)

Vorhalle. Dorische Ordnung. Säulen A 1. A 2 (1:100)

Vorhalle. Oberlager der Säule A 2 (1:50)

Vorhalle. Dorisches Gebälk von Süden

Vorhalle. Dorisches Gebälk von Norden

Vorhalle. Dorisches Gebälk von Osten

Vorhalle. Dorisches Gebälk von Westen

Vorhalle. Dorisches Gebälk. Draufsicht

Vorhalle. Horizontalgeison A 10 (1:50)

Vorhalle. Horizontalgeison A 10

Im Prytaneion deponiertes Horizontalgeison A 11 (1:50)

Im Prytaneion deponiertes Horizontalgeison A 11

Vorhalle. Kapitell A 6

Vorhalle. Kapitell A 5

Vorhalle. Dorische Ordnung mit Lage der Inschriften (1:100)

Vorhalle. Detail Inschriften auf Säule A 3 (IvE 1012. 1020. 1021.

1036. 1040. 1042)

Vorhalle. Säule A 3 Trommel C. Einarbeitung für profilierte Brüstung (1:10) P. M., L. Z., C ÖAI

Vorhalle. Säule A 3 Trommel C. Einarbeitung für profilierte Brüstung

Vorhalle. Säule A 4 Trommel C. Einarbeitung für profilierte Brüstung

Vorhalle. Marmorblock/-platte sowie Sockel der Ehrenbasis SK 15

westlich von Tür 3

Vorhalle. Sockel Ehrenbasis SK 15
B. S., L. Z., (C) ÖAI

M. S., (C) ÖAI

M. S., (C) ÖAI

M. S., (C) ÖAI

M. S., (C) ÖAI

J. E., L. Z., (C) ÖAI

M. S., (C) ÖAI

M. S., (C) ÖAI

M. S., (C) ÖAI

M. S., (C) ÖAI

M. S., (C) ÖAI

M. S., (C) ÖAI

M. S., (C) ÖAI

M. S., (C) ÖAI

J. E., L. Z., (C) ÖAI

M. S., (C) ÖAI

M. S., (C) ÖAI

M. S., (C) ÖAI

M. S., (C) ÖAI

P. M., L. Z., (C) ÖAI

P. M., L. Z., (C) ÖAI

M. S., (C) ÖAI

M. S., (C) ÖAI

P. M., L. Z., (C) ÖAI

P. M., L. Z., (C) ÖAI

M. S., (C) ÖAI

M. S., (C) ÖAI

M. S., (C) ÖAI

M. S., (C) ÖAI

Pl. 494, (C) ÖAI

19563,18 , (C) ÖAI

M. S., (C) ÖAI

M. S., C ÖAI

P. M., L. Z., (C) ÖAI

P. M., L. Z., (C) ÖAI

P. M., L. Z., (C) ÖAI

P. M., L. Z., (C) ÖAI

P. M., L. Z., (C) ÖAI

P. M., L. Z., (C) ÖAI

N. H., L. Z., (C) ÖAI

M. S., (C) ÖAI

M. S., (C) ÖAI

M. S., (C) ÖAI

M. S., (C) ÖAI

N. H., L. Z., (C) ÖAI

M. S., (C) ÖAI

N. H., L. Z., (C) ÖAI

M. S., (C) ÖAI

M. S., (C) ÖAI

M. S., (C) ÖAI

P. M., L. Z., (C) ÖAI

M. S., (C) ÖAI

M. S., (C) ÖAI

M. S., (C) ÖAI

M. S., (C) ÖAI

M. S., (C) ÖAI
N. H., L. Z., C ÖAI

M. S., (C) ÖAI 
Taf. 63, 3:

Taf. 63, 4:

Taf. 63, 5:

Taf. 64, 1:

Taf. 64,2 :

Taf. 65, 1:

Taf. 65, 2:

Taf. 66, 1:

Taf. 66, 2:

Taf. 67, 1:

Taf. 67, 2:

Taf. 68, 1:

Taf. 68, 2:

Taf. 69,1 :

Taf. 69, 2:

Taf. 69, 3:

Taf. 69, 4:

Taf. 69, 5:

Taf. 69, 6:

Taf. 70, 1:

Taf. 70, 2:

Taf. 70, 3:

Taf. 70, 4:

Taf. 71, 1:

Taf. 71, 2:

Taf. 72, 1:

Taf. 72, 2:

Taf. 72, 3:

Taf. 73,1 :

Taf. 73, 2:

Taf. 74,1 :

Taf. 74, 2:

Taf. 75, 1:

Taf. 76, 1:

Taf. 76, 2:

Taf. 77, 1:

Taf. 78, 1:

Taf. 79, 1:

Taf. 80, 1:

Taf. 81, 1:

Taf. 82, 1:

Taf. 83, 1:

Taf. 84,1 :

Taf. 85,1 :

Taf. 86, 1:

Taf. 86, 2:

Taf. 87, 1:

Taf. 87, 2:

Taf. 88,1 :

Taf. 88, 2:

Taf. 89,1 :

Taf. 90, 1:

Taf. 90, 2:

Taf. 91, 1:

Taf. 91, 2:

Taf. 91, 3:
Vorhalle. Sockel Ehrenbasis SK 15 (1:25)

Vorhalle. Marmorplatten östlich von Tür 3

Vorhalle. Marmorspolie mit rinnenförmiger Vertiefung

Vorhalle. Mosaik. Westseite

Vorhalle. Mosaik. Detail Westseite

Vorhalle. Mosaik. Ostseite

>Hestiasaal< von Norden

$>$ Hestiasaalk. Westwand

$>$ Hestiasaak. Nord-Süd-Schnitt nach Westen (1:100)

>Hestiasaal<. Fotogrammetrische Aufnahme der Westwand

>Hestiasaal . Westwand (1:100)

>Hestiasaal<. Südwand

`Hestiasaak. Südwand (1:100)

>Hestiasaal<. Marmornes Türgewände von Tür 2

>Hestiasaal<. Türsturz A 30 in Laibung von Tür 2 verbaut

>Hestiasaalk. Türsturz A 30 in Laibung von Tür 2 verbaut (1:50)

>Hestiasaak. Türsturz A 30 (1:25)

>Hestiasaalk. Türsturz A 31 (1:25)

>Hestiasaak. Türsturz A 31 nach Bergung

>Hestiasaal<. Westliche Türlaibung von Tür 4

$>$ Hestiasaalく. Westliche Türlaibung von Tür 4 (1:50)

>Hestiasaal<. Westliche und östliche Türlaibung von Tür 3

>Hestiasaal<. Westliche und östliche Türlaibung von Tür 3 (1:50)

>Hestiasaak. Ostwand

〉Hestiasaal . Nord-Süd-Schnitt nach Osten (1:100)

$>$ Hestiasaal . Nordostecke. Detail Gesims sowie Rest der Marmorverkleidung

`Hestiasaal<. Fragmente des Gesimses A 26 (1:25)

>Hestiasaalk. Fragmente des Gesimses A 26

$>$ Hestiasaal<. Nordwand

$>$ Hestiasaak. Nordwand (1:100)

`Hestiasaak. Nordwand. Keilsteinbogen Innenansicht

>Hestiasaal<. Nordwand. Keilsteinbogen Draufsicht

>Hestiasaalく. Südwestlicher Säulenstuhl mit Basis A 12. 13

>Hestiasaalく. Südwestlicher Säulenstuhl mit Basis A 12. 13

>Hestiasaal<. Südwestlicher Säulenstuhl mit Basis A 12. 13 (1:50)

>Hestiasaal<. Südöstlicher Säulenstuhl mit Eck-Doppelhalbsäulen A 14-16

>Hestiasaak . Südöstlicher Säulenstuhl mit Eck-Doppelhalbsäulen

A 14-16 (1:50)

>Hestiasaak. Nordöstlicher Säulenstuhl mit Eck-Doppelhalbsäulen A 17-20 M. S., (C ÖAI

>Hestiasaal<. Nordöstlicher Säulenstuhl mit Eck-Doppelhalbsäulen A 17-20 J. E., P. M., L. Z., C ÖAI $(1: 50)$

>Hestiasaak. Detail Kompositkapitell A 20

`Hestiasaal<. Nordwestlicher Säulenstuhl mit Eck-Doppelhalbsäulen

A 21-24

>Hestiasaak. Nordwestlicher Säulenstuhl mit Eck-Doppelhalbsäulen

A $21-24(1: 50)$

>Hestiasaal<. Detail Kompositkapitell A 24

>Hestiasaal<. Detail Kompositkapitell A 24

>Hestiasaak. Originale attische Basis A 47

>Hestiasaal<. Originale attische Basis A 47 (1:25)

>Hestiasaal<. Kompositkapitell A 25

>Hestiasaal<. Kompositkapitell A 25 (1:25)

>Hestiasaal<. Werkriss auf südwestlichem Säulenstuhl A 12 (Volute 1)

$>$ Hestiasaalく. Werkriss auf südwestlichem Säulenstuhl A 12 (Volute 2)

>Hestiasaalく. Werkriss auf südwestlichem Säulenstuhl A 12 (1:4)

`Hestiasaak. Zentrales Fundament für Anrichte

`Hestiasaal . Zentrales Fundament für Anrichte. Freigelegte Westseite mit Versatzmarken zur Verlegung der Blöcke

`Hestiasaal<. Unterkonstruktion für Sitzbänke an der Ostseite von Norden >Hestiasaal . Unterkonstruktion für Sitzbänke an der Westseite von Süden `Hestiasaalく Unterkonstruktion für Sitzbänke an der Westseite von Norden
B. S., L. Z., (C) ÖAI

M. S., C ÖAI

M. S., (C) ÖAI

M. S., (C) ÖAI

M. S., C ÖAI

M. S., (C) ÖAI

M. S., (C) ÖAI

M. S., (C) ÖAI

P. M., J. E., L. Z., (C) ÖAI

C. K.

P. M., L. Z., (C) ÖAI

M. S., (C) ÖAI

P. M., L. Z., (C) ÖAI

M. S., (C) ÖAI

M. S., C ÖAI

J. E., L. Z., C ÖAI

B. S., L. Z., (C) ÖAI

B. S., L. Z., (C) ÖAI

M. S., (C) ÖAI

M. S., (C) ÖAI

J. E., L. Z., (C) ÖAI

M. S., (C) ÖAI

J. E., L. Z., (C) ÖAI

M. S., (C) ÖAI

P. M., J. E., L. Z., (C) ÖAI

N. H., L. Z., (C) ÖAI

M. S., (C) ÖAI

M. S., (C) ÖAI

P. M., L. Z., (C) ÖAI

M. S., (C) ÖAI

M. S., (C) ÖAI

M. S., (C) ÖAI

M. S., (C) ÖAI

J. E., L. Z., (C) ÖAI

M. S., (C) ÖAI

J. E., L. Z., C ÖAI

M. S., (C) ÖAI

M. S., (C) ÖAI

J. E., P. M., L. Z., (C) ÖAI

M. S., (C) ÖAI

M. S., C ÖAI

M. S., (C) ÖAI

P. M., L. Z., (C) ÖAI

M. S., (C) ÖAI

N. H., L. Z., (C) ÖAI

N. G., (C) ÖAI

N. G., (C) ÖAI

N. H., M. S., L. Z., (C) ÖAI

M. S., (C) ÖAI

M. S., (C) ÖAI

M. S., (C) ÖAI

M. S., (C) ÖAI

M. S., (C) ÖAI 
Taf. 92, 1:

Taf. 92, 2:

Taf. 93, 1:

Taf. 93, 2:

Taf. 94, 1:

Taf. 94, 2:

Taf. 95, 1:

Taf. 96, 1:

Taf. 97, 1:

Taf. 97, 2:

Taf. 98, 1:

Taf. 98, 2:

Taf. 99, 1:

Taf. 99, 2:

Taf. 100,1 :

Taf. 100, 2:

Taf. 101, 1:

Taf. 101, 2:

Taf. 102, 1:

Taf. 102, 2:

Taf. 103, 1:

Taf. 103, 2:

Taf. 104, 1:

Taf. 104, 2:

Taf. 105, 1:

Taf. 106, 1:

Taf. 106, 2:

Taf. 107, 1:

Taf. 107, 2:

Taf. 108, 1:

Taf. 109, 1:

Taf. 110, 1:

Taf. 110, 2:

Taf. 111, 1:

Taf. 112, 1:

Taf. 112, 2:

Taf. 113, 1:

Taf. 113, 2:

Taf. 114, 1:

Taf. 114, 2:

Taf. 114, 3:

Taf. 115,1 :

Taf. 115, 2:

Taf. 115, 3:

Taf. 116, 1:

Taf. 116, 2:

Taf. 117, 1:

Taf. 117, 2:

Taf. 118, 1:

Taf. 119, 1:

Taf. 120, 1:

Taf. 120, 2:

Taf. 120, 3:

Taf. 121, 1:

Taf. 121, 2:

Taf. 122, 1:

Taf. 122, 2:

Taf. 123, 1:

Taf. 124, 1:

Taf. 124, 2:

Taf. 125, 1:
Raum 6 von Norden

Raum 6. Südwand (1:50)

M. S., (C) ÖAI

Raum 6/Vorhalle. Tür 1. Westliche Türlaibung und östliche Zusetzung (1:50)

Raum 6. Nordwand. Detail Wandmalerei

Raum 6. Ostwand

Raum 6. Westwand

Raum 6. Ostwand (1:50)

Raum 6. Westwand (1:50)

Raum 6. Nordwand

Raum 6. Nordwand (1:50)

Raum 6/Raum 5. Tür von Norden

Raum 6/Raum 5. Westliche und östliche Türlaibung (1:50)

Raum 6. Säulentrommel A 29

Raum 6. Säulentrommel A 29 (1:25)

Raum 6. Rampenartige Treppe an der Südwand

Raum 6. Marmorbasis vor der Ostwand

Raum 6. An die Nordwand angesetzte sekundäre Mauer

Raum 6. Detail der an die Nordwand angesetzten sekundären Mauer

Raum 5 von Süden

Raum 5 von Norden

Raum 5. Südwand

Raum 5. Südwand (1:50)

Raum 5. Westwand

Raum 5/>Akademiegasseく. Marmorschwelle der sekundären Tür

Raum 5. Westwand (1:50)

Raum 5. An die Nordwand angesetzte sekundäre Mauer

Raum 5. Detail der an die Nordwand angesetzten sekundären Mauer

Raum 5. Ostwand

Raum 5. Nordwand

Raum 5. Ostwand (1:50)

Raum 5. Nordwand (1:50)

Raum 5. Sekundäre Nord-Süd-Mauer von Westen

Raum 5. Sekundäre Nord-Süd-Mauer von Osten

Raum 5. Sekundäre Nord-Süd-Mauer (1:50)

Raum 5. Sekundäre Nord-Süd-Mauer vor Sicherung

Raum 5. Gewölbe vor Absturz der Ostwand

Raum 5. Sekundäre Mauer zwischen Säule und Westwand

Raum 5. Säule A 27. 28 (1:50)

Kanal nördlich der Räume 5, 4, 3, 2

Bauwich nördlich der Räume 5, 4, 3, 2

Kanal nördlich der Räume 5, 4, 3, 2. Schnitt nach Osten (1:50)

Raum 4 von Norden

Raum 4. Nordwand

Raum 4. Nordwand (1:50)

Raum 4. Südwand

Raum 4. Südwand (1:50)

Raum 4. Westwand

Raum 4. Westwand (1:50)

Raum 4. Pfeiler der Ostwand

Raum 4. Pfeiler der Ostwand (1:50)

Raum 3 von Osten

Raum 3. Südwand

Raum 3. Südwand (1:50)

Raum 3. Pfeiler der Westwand

Raum 3. Pfeiler der Westwand (1:50)

Raum 3. Nordwand oberer Teil

Raum 3. Nordwand unterer Teil

Raum 3. Nordwand (1:50)

Raum 3. Ostwand

Raum 3. Ostwand (1:50)

Raum 3. Ausfluss des Wasserreservoirs
P. M., L. Z., (C) ÖAI

J. E., L. Z., (C) ÖAI

M. S., (C) ÖAI

M. S., (C) ÖAI

M. S., (C) ÖAI

P. M., L. Z., (C) ÖAI

P. M., L. Z., (C) ÖAI

M. S., (C) ÖAI

P. M., L. Z., (C) ÖAI

M. S., (C) ÖAI

J. E., L. Z., (C) ÖAI

M. S., (C) ÖAI

N. H., L. Z., (C) ÖAI

1961252,8 , (C) ÖAI

1961251,11 , C ÖAI

1961251,5 , (C) ÖAI

1961 254, 1, C ÖAI

M. S., (C) ÖAI

M. S., (C) ÖAI

M. S., (C) ÖAI

P. M., L. Z., (C) ÖAI

M. S., (C) ÖAI

M. S., (C) ÖAI

P. M., L. Z., (C) ÖAI

P1. 561, (C) ÖAI

Pl. 576, (C) ÖAI

M. S., (C) ÖAI

M. S., (C) ÖAI

P. M., L. Z., (C) ÖAI

P. M., L. Z., (C) ÖAI

M. S., (C) ÖAI

M. S., (C) ÖAI

P. M., L. Z., (C) ÖAI

1961 252, 12, (C) ÖAI

Pl. 533, (C) ÖAI

Pl. 602, (C) ÖAI

N. H., L. Z., (C) ÖAI

M. S., (C) ÖAI

M. S., (C) ÖAI

N. H., L. Z., (C) ÖAI

M. S., (C) ÖAI

M. S., (C) ÖAI

P. M., L. Z., (C) ÖAI

M. S., (C) ÖAI

P. M., L. Z., (C) ÖAI

M. S., (C) ÖAI

P. M., L. Z., (C) ÖAI

M. S., (C) ÖAI

P. M., L. Z., (C) ÖAI

M. S., (C) ÖAI

M. S., (C) ÖAI

P. M., L. Z., (C) ÖAI

M. S., (C) ÖAI

P. M., L. Z., C ÖAI

M. S., (C) ÖAI

M. S., (C) ÖAI

P. M., L. Z., (C) ÖAI

M. S., (C) ÖAI

P. M., L. Z., (C) ÖAI

M. S., (C) ÖAI 
Taf. 125, 2: Taf. 125, 3: Taf. 126, 1: Taf. 126, 2: Taf. 127, 1: Taf. 127, 2: Taf. 128,1 : Taf. 128, 2: Taf. 129, 1: Taf. 129, 2: Taf. 130, 1: Taf. 130, 2: Taf. 131, 1: Taf. 131, 2: Taf. 131, 3: Taf. 131, 4: Taf. 131, 5: Taf. 131, 6: Taf. 132, 1: Taf. 132, 2: Taf. 132, 3: Taf. 132, 4: Taf. 132, 5: Taf. 132, 6: Taf. 133, 1: Taf. 133, 2: Taf. 133, 3: Taf. 133, 4: Taf. 133, 5: Taf. 133, 6: Taf. 134, 1: Taf. 134, 2: Taf. 134, 3: Taf. 134, 4: Taf. 134, 5: Taf. 134, 6: Taf. 135, 1: Taf. 135, 2: Taf. 135, 3: Taf. 135, 4: Taf. 136, 1: Taf. 136, 2: Taf. 136, 3: Taf. 136, 4:

Kapitel IV Taf. 137, 1: Taf. 138, 1: Taf. 138, 2: Taf. 139, 1: Taf. 139, 2: Taf. 139, 3: Taf. 140, 1: Taf. 140, 2: Taf. 141, 1: Taf. 141, 2: Taf. 141, 3: Taf. 142, 1: Taf. 142, 2: Taf. 142, 3: Taf. 143, 1:
>Hestiasaal<. Sekundäre Öffnung in Nordwand zur Wasserentnahme

Raum 2 von Westen

Raum 2. Südwand

Raum 2. Südwand (1:50)

Raum 2. Westwand

Raum 2. Westwand (1:50)

Raum 2. Nordwand oberer Teil

Raum 2. Nordwand (1:50)

Raum 2. Ostwand

Raum 2. Ostwand (1:50)

Raum 2. Ziegelbogen in Sturzlage

Raum 2. Ende der 1960er Jahre bereits weitgehend zerstörter Ziegelbogen Attische Basis A 32

Attische Basis A 32 (1:25)

Attische Basis A 33

Attische Basis A 33 (1:25)

Attische Basis A 34

Attische Basis A 34 (1:25)

Attische Basis A 35

Attische Basis A 35 (1:25)

Attische Basis einer Dreiviertelsäule A 36

Attische Basis einer Dreiviertelsäule A 36 (1:25)

Rundbasis A 37

Rundbasis A 37 (1:25)

Pfeiler-/Pilasterbasis A 38

Pfeiler-/Pilasterbasis A 38 (1:25)

Pfeiler-/Pilasterbasis A 39

Pfeiler-/Pilasterbasis A 39 (1:25)

Pfeiler A 40

Pfeiler A 40 (1:25)

Zahnschnittgeison mit Sima A 41

Zahnschnittgeison mit Sima A 41 (1:25)

Dorisches Kapitell A 42

Dorisches Kapitell A 42 (1:25)

Tuskanisches Kapitell A 43

Tuskanisches Kapitell A 43 (1:25)

Korinthisches Kapitell A 44

Korinthisches Kapitell A 44 (1:25)

Pilasterkapitell A 45

Pilasterkapitell A 45 (1:25)

Kämpfer A 46

Kämpfer A 46 (1:25)

Sockel Ehrenbasis? SK 16

Sockel Ehrenbasis? SK 16 (1:25)
M. S., (C) ÖAI

M. S., (C) ÖAI

M. S., (C) ÖAI

P. M., L. Z., (C) ÖAI

M. S., (C) ÖAI

P. M., L. Z., (C) ÖAI

M. S., (C) ÖAI

P. M., L. Z., C ÖAI

M. S., (C) ÖAI

P. M., L. Z., (C) ÖAI

Pl. 529, (C) ÖAI

1967 573, 27, C ÖAI

M. S., (C) ÖAI

B. S., L. Z., (C) ÖAI

M. S., (C) ÖAI

B. S., L. Z., (C) ÖAI

M. S., (C) ÖAI

B. S., L. Z., (C) ÖAI

M. S., (C) ÖAI

B. S., L. Z., ( $\odot$ ÖAI

M. S., C ÖAI

B. S., L. Z., (C) ÖAI

M. S., (C) ÖAI

B. S., L. Z., (C) ÖAI

M. S., (C) ÖAI

N. H., L. Z., (C) ÖAI

M. S., (C) ÖAI

B. S., L. Z., (C) ÖAI

M. S., (C) ÖAI

B. S., L. Z., (C) ÖAI

M. S., (C) ÖAI

B. S., L. Z., (C) ÖAI

M. S., (C) ÖAI

N. H., L. Z., C ÖAI

M. S., (C) ÖAI

B. S., L. Z., ( $)$ ÖAI

M. S., (C) ÖAI

N. H., L. Z., (C) ÖAI

M. S., (C) ÖAI

B. S., L. Z., (C) ÖAI

M. S., (C) ÖAI

B. S., L. Z., (C) ÖAI

M. S., (C) ÖAI

B. S., L. Z., (C) ÖAI

Prytaneion. Generalisierter Grundriss mit Sondagenplan 2007-2008 (1:250)

Schnitt 1/07. Ausgangssituation von Süden

Schnitt 1/07. Endsituation von Süden

Schnitt 1/07. Endsituation von Westen

Schnitt 1/07. Westprofil

Schnitt 1/07. Nordprofil

Schnitt 1/07. Westprofil (1:50)

Schnitt 1/07. Nordprofil (1:50)

Schnitt 2/07. Ausgangssituation von Süden

Schnitt 2/07. Kanal SE 204

Schnitt 2/07. Endsituation von Süden

Schnitt 2/07. Südprofil

Schnitt 2/07. Ostprofil

Schnitt 2/07. Nordprofil

Schnitt 2/07. Südprofil (1:50) 
Taf. 143, 2: Taf. 144, 1: Taf. 144, 2:

Taf. 145,1 : Taf. 145, 2: Taf. 146, 1: Taf. 146, 2: Taf. 147, 1: Taf. 147, 2:

Taf. 147, 3: Taf. 148, 1: Taf. 148, 2: Taf. 148, 3: Taf. 149, 1: Taf. 149, 2: Taf. 149, 3: Taf. 150, 1: Taf. 150, 2: Taf. 151, 1: Taf. 151, 2: Taf. 152, 1: Taf. 153, 1: Taf. 153, 2: Taf. 154, 1: Taf. 155, 1: Taf. 155, 2: Taf. 156, 1: Taf. 156, 2: Taf. 157, 1: Taf. 157, 2: Taf. 158, 1: Taf. 158, 2:

Kapitel V Taf. 159, 1:

Taf. 160, 1:

Taf. 161, 1:

Kapitel VI

Taf. 162:

Taf. 163:

Taf. 164:

Taf. 165:

Taf. 166:

Taf. 167:

Taf. 168:

Taf. 169:

Taf. 170:

Taf. 171:

Taf. 172:

Taf. 173:

Taf. 174:

Taf. 175:

Taf. 176:

Taf. 177:

Taf. 178:
Schnitt 2/07. Nordprofil (1:50)

Schnitt 3/07. Ausgangssituation von Süden

A. H., L. Z., C ÖAI

$\begin{array}{ll}\text { Schnitt 3/07. Ausgangssituation von Süden } & \text { M. S., (C) ÖAI } \\ \text { Schnitt 3/07. Fundament und Estrich (SE 303, SE 304) des Marmorplatten- } & \text { M. S., (C) ÖAI }\end{array}$ bodens

Schnitt 3/07. Endsituation von Süden

Schnitt 3/07. Planum Endzustand (1:50)

Schnitt 3/07. Ostprofil

Schnitt 3/07. Ostprofil (1:50)

Schnitt 4/07. Ausgangssituation von Osten

Schnitt 4/07. Fundament und Estrich (SE 402 bodens

Schnitt 4/07. Kalkgrube SE 407

Schnitt 4/07. Endsituation von Osten

Schnitt 4/07. Ostprofil

Schnitt 4/07. Südprofil

Schnitt 4/07. Nordprofil (1:20)

Schnitt 4/07. Ostprofil $(1: 20)$

Schnitt 4/07. Südprofil $(1: 20)$

Schnitt 5/07. Ausgangssituation von Westen

Schnitt 5/07. Endsituation von Westen

Schnitt 5/07. Endsituation von Süden

Schnitt 5/07. Nordprofil

Schnitt 5/07. Süd- und Nordprofil (1:20)

Schnitt 1/08. Ausgangssituation von Westen

Schnitt 1/08. Endsituation von Westen

Schnitt 1/08. Planum Endzustand (1:50)

Schnitt 1/08. Nordprofil

Schnitt 1/08. Nordprofil $(1: 20)$

Schnitt 1/08. Ostprofil

Schnitt 1/08. Ostprofil (1:20)

Schnitt 1/08. Westprofil

Schnitt 1/08. Westprofil $(1: 20)$

Sekundäres Wasserreservoir in Raum 7 östlich des Prytaneions

Sekundäre Wasserentnahmestelle östlich von Raum 7

M. S., (C) ÖAI

B. S., L. Z., (C) ÖAI

M. S., (C) ÖAI

B. S., L. Z., C ÖAI

M. S., C ÖAI

M. S., (C) ÖAI

M. S., (C) ÖAI

M. S., (C) ÖAI

M. S., (C) ÖAI

M. S., (C) ÖAI

B. S., L. Z., (C) ÖAI

B. S., L. Z., (C) ÖAI

B. S., L. Z., (C) ÖAI

M. S., (C) ÖAI

M. S., (C) ÖAI

M. S., (C) ÖAI

M. S., (C) ÖAI

B. S., L. Z., (C) ÖAI

M. S., (C) ÖAI

M. S., (C) ÖAI

B. S., L. Z., (C) ÖAI

M. S., (C) ÖAI

B. S., L. Z., (C) ÖAI

M. S., (C) ÖAI

B. S., L. Z., (C) ÖAI

M. S., (C) ÖAI

B. S., L. Z., (C) ÖAI

M. S., (C) ÖAI

M. S., (C) ÖAI

L. Z., M. S., P. M., (C) ÖAI

L. Z., M. S., P. M., C ÖAI

L. Z., M. S., P. M., (C ÖAI

N. H., N. M., (C) ÖAI

N. H., N. M., (C) ÖAI

N. H., N. M., (C) ÖAI

N. H., N. M., (C) ÖAI

N. H., N. M., (C) ÖAI

N. H., N. M., (C) ÖAI

N. H., N. M., () ÖAI

N. H., N. M., C ÖAI

N. H., N. M., (C) ÖAI

N. H., N. M., (C) ÖAI

N. H., N. M., (C) ÖAI

N. H., N. M., (C) ÖAI

N. H., N. M., (C) ÖAI

N. H., N. M., (C) ÖAI

N. H., N. M., (C) ÖAI

N. H., N. M., (C) ÖAI

N. H., N. M., (C) ÖAI 
Taf. 179:

Taf. 180:

Taf. 181:

Taf. 182:

Taf. 183:

Taf. 184:

Taf. 185:

Taf. 186:

Taf. 187:

Taf. 188:

Taf. 189:

Taf. 190:

Taf. 191:

Taf. 192:

Taf. 193:

Taf. 194:

Taf. 195:

Taf. 196:

Taf. 197:

Taf. 198:

Taf. 199:

Taf. 200:

Taf. 201:

Taf. 202:

Taf. 203:

Taf. 204:

Taf. 205:

Taf. 206:

Taf. 207:

Taf. 208:

Taf. 209:

Taf. 210:

Taf. 211:

Taf. 212:

Taf. 213:

Taf. 214:

Taf. 215:

Taf. 216:

Taf. 217:

Taf. 218:

Taf. 219:

Taf. 220:

Taf. 221:

Taf. 222:

Taf. 223:

Taf. 224:

Taf. 225:

Kapitel VII

Taf. 226, 1:

Taf. 226, 2:

Taf. 227, 1:

Taf. 227, 2:

Taf. 228, 1-3:

Taf. 229, 1:

Taf. 229, 2:

Taf. 229, 3:

Taf. 230, 1. 2:

Taf. 231, 1:

Taf. 231, 2:

Taf. 231, 3:
K 261-275

K 276-282. 285-294

K 295-301. 304-310. 313-318

K 319-335

K 336-356

K 357-372

K 373-390

K 391-402

K 403-414

K 415-428

K 429-438

K 439-448

K 449-458

K 459-466. 468-472

K 473-483

K 484-497

K 498-510

K 511-520

K 521-528

K 529-536

K 537-545

K 546-557

K 558-571

K 572-589

K 590-603

K 604-617

K 618-622. 624-626

K 627-637

K 638. 640-658

K 659-673

K 674-686

K 687-697

K 698-704. 706-708. 712-714

K 715-730

K 731-740

K 741-752

K 753-766

K 767-779

K 781-786. 788-794

K 4. 36. 37. 60. 89. 116. 120.121. 132. 134. 139-143

K 144-150. 158. 162. 164-166. 173

K 214. 226. 279-286

K 287. 288. 291. 302. 303. 311. 312

K 321. 355. 428. 462. 467. 488. 529

K 616-622

K 638.639.641. 647.649.652.674.705.708-711

K 751. 787-789. 792

$>$ Neronische Halle $<$. Blick von Norden

$>$ Neronische Halle . Sockelzone an der >Marmorstraße

`Neronische Halleく. Polsterquader der Sockelzone: Saum an den Stoßflächen >Neronische Halleく. Stylobat mit Risslinien zur Markierung der Säulenachse >Neronische Halle‘. Dorische Kapitelle

$>$ Neronische Halle<. Eckarchitrav

$>$ Neronische Halle . Architrav: Soffitte

$>$ Neronische Halle`. Architrav: Rückseite

N. H., N. M., (C) ÖAI

N. H., N. M., (C) ÖAI

N. H., N. M., (C) ÖAI

N. H., N. M., (C) ÖAI

N. H., N. M., (C) ÖAI

N. H., N. M., (C) ÖAI

N. H., N. M., (C) ÖAI

N. H., N. M., (C) ÖAI

N. H., N. M., (C) ÖAI

N. H., N. M., (C) ÖAI

N. H., N. M., (C) ÖAI

N. H., N. M., (C) ÖAI

N. H., N. M., (C) ÖAI

N. H., N. M., (C) ÖAI

N. H., N. M., (C) ÖAI

N. H., N. M., (C) ÖAI

N. H., N. M., (C) ÖAI

N. H., N. M., (C) ÖAI

N. H., N. M., (C) ÖAI

N. H., N. M., (C) ÖAI

N. H., N. M., C ÖAI

N. H., N. M., (C) ÖAI

N. H., N. M., (C) ÖAI

N. H., N. M., (C) ÖAI

N. H., N. M., (C) ÖAI

N. H., N. M., (c) ÖAI

N. H., N. M., (C) ÖAI

N. H., N. M., (C) ÖAI

N. H., N. M., (C) ÖAI

N. H., N. M., (C) ÖAI

N. H., N. M., (C) ÖAI

N. H., N. M., (C) ÖAI

N. H., N. M., (C) ÖAI

N. H., N. M., (C) ÖAI

N. H., N. M., (C) ÖAI

N. H., N. M., (C) ÖAI

N. H., N. M., (C) ÖAI

N. H., N. M., (C) ÖAI

N. H., N. M., (C) ÖAI

N. G., (C) ÖAI

N. G., (C) ÖAI

N. G., (C) ÖAI

N. G., (C) ÖAI

N. G., (C) ÖAI

N. G., (C) ÖAI

N. G., (C) ÖAI

N. G., (C) ÖAI

G. P.

G. P.

G. P.

G. P.

G. P.

G. P.

G. P.

G. P.

G. P.

G. P.

G. P.

G. P.
>Neronische Halle<. Geison

`Neronische Halle`. Geison: Wasserrinne an der Oberseite

>Neronische Halleく. Geison: vertikaler Gusskanal an der Stoßfuge 
Taf. 232, 1: Taf. 232, 2:

Taf. 233, 1:

Taf. 233, 2:

Taf. 234, 1:

Taf. 235, 1 :

Taf. 235, 2:

Taf. 235, 3:

Taf. 236, 1 :

Taf. 236, 2:

Taf. 237, 1. 2:

Taf. 238, 1:

Taf. 238, 2:

Taf. 238, 3:

Taf. 239, 1:

Taf. 239, 2:

Taf. 239, 3:

Taf. 240, 1:

Taf. 240, 2:

Taf. 240, 3 :

Taf. 240, 4:

Kapitel IX

Taf. 241, 1:

Taf. 241, 2:

Taf. 242, 1:

Taf. 242, 2:

Taf. 243, 1:

Taf. 243, 2:

Taf. 243, 3:

Taf. 244, 1:

Taf. 244, 2:

Taf. 244, 3:

Taf. 245, 1:

Taf. 245, 2 :

Taf. 246, 1:

Taf. 246, 2:

Taf. 247, 1:

Taf. 247, 2:

Taf. 247, 3:

Taf. 248, 1:

Taf. 248, 2:

Taf. 248, 3:

Schnitte

Taf. 249, 1:
`Neronische Halleく. Geison: Südostecke mit Ansatz des Schräggeisons

$>$ Neronische Halleく. Eckgeison von der Nordseite

$>$ Neronische Halle<. Zugang an der östlichen Langseite

>Neronische Halle<. Dorisches >Antenkapitell am Zugang in der

östlichen Langseite

Domitiansterrasse. Architekturprobe der Fassade

Domitiansterrasse. Dorisierende Kapitelle vom Untergeschoss der Fassade

Domitiansterrasse. Dorisches Gebälk des Untergeschosses der Fassade

Domitiansterrasse. Sockelzone des mittleren Fassadengeschosses

Vediusgymnasium. Pfeilerkapitell aus dem $>$ Marmorsaak

Ostgymnasium. Pfeilerkapitell aus dem >Marmorsaal

〉Südliches Hafentor<. Pfeilerkapitell

Tetragonos Agora. Architrav

Basilike Stoa auf dem >Staatsmarkt $<$. Halbsäulen-Pfeilerkapitell

vom Obergeschoss

Domitiansplatz. Kapitell

Männlicher Porträtkopf SK 5

Porträt einer jungen Frau SK 6

Porträt einer Frau SK 7

Männliche Porträtbüste SK 8

Männliche, hermenförmige Büste SK 9

Linker Unterarm einer männlichen Kolossalstatue SK 11

Torso einer männlichen Ehrenstatue SK 10

Bouleuterion von Aigai

Übersichtsplan der Agora von Herakleia am Latmos

Nördliche Stoa der Agora von Kolophon

Übersichtsplan der Agora von Magnesia am Mäander

Stadtplan von Milet

Sog. Prytaneion von Milet in der Südwestecke des Nordmarktes von Süden Delphinion von Milet

Bau H von Pergamon

Bau $\mathrm{H}$ von Pergamon von Westen

Bau $\mathrm{H}$ von Pergamon von Nordosten

Grundriss des Prytaneions von Priene

Prytaneion von Priene von Nordwesten

Prytaneion von Priene von Osten

Prytaneion von Priene. Ehreninschrift für M. Aurelius Tatianus

Prytaneion von Delos von Süden

Prytaneion von Lato auf Kreta von Südwesten

Aufgang zum Prytaneion von Lato von Süden

Prytaneion von Dodona von Nordwesten

Grundriss des Prytaneions von Kassope

Tholos der Athener Agora von Südosten

Prytaneion. Übersicht Schnitte durch Bauaufnahme (1:250)
G. P.

G. P.

G. P.

G. P.

G. P.

G. P.

G. P.

G. P.

G. P.

G. P.

G. P.

G. P.

G. P.

G. P.

N. G., (C) ÖAI

N. G., (C) ÖAI

N. G., (C) ÖAI

N. G., (C) ÖAI

N. G., (C) ÖAI

(C) ÖAI

N. G., (C) ÖAI

M. S.

K. Wulzinger, Das Rathaus von Herakleia am Latmos, in: F. Krischen, Antike Rathäuser (Berlin 1941) Taf. 26.

Holland 1944, Abb. 10.

Humann 1904, Blatt 3.

Herda 2005, Abb. 1 (nach

B. F. Weber, Ausschnitt).

M. S.

M. S.

W. Dörpfeld, Die Arbeiten zu Pergamon 1908-1909, AM 35, 1910, Taf. 15 (Ausschnitt).

M. S.

M. S.

Th. Wiegand - H. Schrader, Priene. Ergebnisse der Ausgra bungen und Untersuchungen in den Jahren 1895-1898 (Berlin 1904) Abb. 225.

M. S.

M. S.

M. S.

R. Étienne, Le Prytanée de Délos, REA 99, 1997, Abb. 2.

M. S.

M. S.

M. S.

Hoepfner - Schwandner 1986, Abb. 68.

M. S.

L. Z., P. M., M. S., J. E., (C) ÖAI 
Taf. 250, 1:

Taf. 251, 1:

Taf. 252, 1:

Taf. 253, 1:

Taf. 254, 1:

Taf. 254, 2:

Taf. 255, 1:

Taf. 256, 1:

Taf. 256, 2:

Taf. 257, 1:

Taf. 258, 1:

3-D-Modelle

Taf. 259, 1:

Taf. 260, 1:

Taf. 261, 1:

Taf. 262, 1:

Taf. 263, 1:
Prytaneion. Schnitt A-A' (1:100)

Prytaneion. Schnitt B-B' $(1: 125)$

Prytaneion. Schnitt C-C' $(1: 100)$

Prytaneion. Schnitt D-D' $(1: 100)$

Prytaneion. Schnitt E-E' $(1: 150)$

Prytaneion. Schnitt F-F' (1:150)

Prytaneion. Übersicht Rekonstruktionsschnitte (1:250)

Prytaneion. Schnitt a-a' (1:150)

Prytaneion. Schnitt b-b' (1:150)

Prytaneion. Schnitt c-c' (1:100)

Prytaneion. Schnitt d-d' (1:100)

Prytaneion. Geschlossenes Modell von Südosten

Prytaneion. Geöffnetes Modell von Südosten

Prytaneion. Vogelperspektive des Modells von Südwesten

Prytaneion. Modellperspektive vom Vorhof in die Vorhalle

Prytaneion. Modellperspektive des \Hestiasaales` Richtung Norden
L. Z., P. M., (C) ÖAI

L. Z., P. M., N. H., (C) ÖAI

L. Z., P. M., (C) ÖAI

L. Z., P. M., A. H., (C) ÖAI

L. Z., P. M., N. H., J. E., (C)

ÖAI

L. Z., P. M., N. H., J. E., (C)

ÖAI

L. Z., P. M., M. S., C ÖAI

M. L., (C) ÖAI

M. L., (C) ÖAI

M. L., (C) ÖAI

M. L., (C) ÖAI

M. L., J. J., (C) ÖAI

M. L., J. J., (C) ÖAI

M. L., J. J., (C) ÖAI

M. L., J. J., (C) ÖAI

M. L., J. J., (C) ÖAI

\section{TextabBildungen}

\begin{tabular}{|c|c|c|c|}
\hline Textabb. & Seite & Inhalt & Nachweis \\
\hline Abb. 1: & 66 & Harris Matrix. Schnitt 1/07 & M. S., (C) ÖAI \\
\hline Abb. 2: & 68 & Harris Matrix. Schnitt 2/07 & M. S., (C) ÖAI \\
\hline Abb. 3: & 71 & Harris Matrix. Schnitt 3/07 & M. S., C ÖAI \\
\hline Abb. 4: & 72 & Harris Matrix. Schnitt 4/07 & M. S., (C) ÖAI \\
\hline Abb. 5: & 73 & Harris Matrix. Schnitt 5/07 & M. S., (C) ÖAI \\
\hline Abb. 6: & 75 & Harris Matrix. Schnitt 1/08 & M. S., (C) ÖAI \\
\hline Abb. 7: & 86 & Scherbenzahl pro stratigrafische Einheit & S. L., C ÖAI \\
\hline Abb. 8: & 86 & Scherbenzahl pro Bauphase & S. L., (C) ÖAI \\
\hline Abb. 9: & 87 & $\begin{array}{l}\text { Sigillataspektrum aus den Grabungen im Prytaneion 2007/2008 } \\
\text { (blau: Randstücke, rot: Bodenstücke, gelb: Wandstücke) }\end{array}$ & S. L., C ÖAI \\
\hline Abb. 10: & 88 & $\begin{array}{l}\text { Zusammensetzung des Fundspektrums des hellenistischen } \\
\text { Terrassierungsstratums }\end{array}$ & S. L., C ÖAI \\
\hline Abb. 11: & 89 & $\begin{array}{l}\text { Zusammensetzung der Feinware des hellenistischen } \\
\text { Terrassierungsstratums }\end{array}$ & S. L., (C) ÖAI \\
\hline Abb. 12: & 91 & Zusammensetzung des Fundspektrums von Bauphase 1 & S. L., C ÖAI \\
\hline Abb. 13: & 91 & Zusammensetzung der Sigillaten von Bauphase 1 & S. L., C ÖAI \\
\hline Abb. 14: & 91 & Zusammensetzung der hellenistischen Feinware von Bauphase 1 & S. L., C ÖAI \\
\hline
\end{tabular}


